\title{
Endoscopic orbital fat decompression for the management of proptosis in Grave's orbitopathy using a laryngeal skimmer blade
}

\author{
David S Curragh ${ }^{1,2} \cdot$ Dinesh Selva ${ }^{1,2}$
}

Received: 31 October 2018 / Revised: 17 April 2019 / Accepted: 21 June 2019 / Published online: 8 July 2019

(c) The Author(s), under exclusive licence to The Royal College of Ophthalmologists 2019

\begin{abstract}
Backgrounds/objectives Intraoperative handling and manipulation of orbital fat remains a challenge to orbital surgeons. We present a case series of endoscopic orbital fat decompression with medial orbital wall decompression for proptosis management in Grave's orbitopathy, describing a technique for fat excision using a laryngeal skimmer blade, reporting clinical and surgical outcomes, and complications.

Subjects/methods All patients who underwent endoscopic orbital fat decompression, with medial orbital wall decompression, for proptosis management in Grave's orbitopathy between 2011 and 2018, under the care of a single surgeon, were included in this retrospective interventional case series.

Results Nineteen patients were included in this study. Using a laryngeal skimmer blade, orbital fat was excised endoscopically at the time of medial orbital wall decompression. The mean volume of orbital fat excised was $1.45 \pm 0.63 \mathrm{ml}$ and the mean reduction in proptosis was $4.5 \pm 1.02 \mathrm{~mm}$. There were no intraoperative complications.

Conclusions In this study, we describe our experience of using a laryngeal skimmer blade as a method of excising orbital fat for orbital decompression in patients with proptosis secondary to Grave's orbitopathy.
\end{abstract}

\section{Introduction}

Intraoperative handling and manipulation of orbital fat remains a challenge to orbital surgeons. The role of orbital fat is to provide mechanical support to the globe [1]. However, it can become involved in various infiltrative and inflammatory orbital diseases including proliferation in thyroid eye disease [2]. Excising orbital fat can be an adjunct to bony decompression in Graves orbitopathy and can be performed for visual reasons, in the case of dysthyroid optic neuropathy, or for proptosis [3-9]. Some debate exists regarding how much proptosis reduction can be achieved by excising orbital fat alone, or in conjunction with medial orbital wall decompression and there is also concern about post-operative diplopia when not balanced

David S Curragh

davidcurragh@hotmail.com

1 Department of Ophthalmology \& Visual Sciences, University of Adelaide, North Terrace, SA 5000, Australia

2 South Australian Institute of Ophthalmology, Royal Adelaide Hospital, Port Road, SA 5000, Australia with a lateral orbital wall decompression $[5,10]$. We present a case series of endoscopic orbital fat decompression with medial orbital wall decompression for proptosis management in Grave's orbitopathy, describing a technique for fat excision using a laryngeal skimmer blade, reporting clinical and surgical outcomes, and complications.

\section{Materials and methods}

All patients who underwent endoscopic orbital fat decompression, with medial orbital wall decompression, for proptosis management in Grave's orbitopathy between January 2011 and September 2018, under the care of a single surgeon (DS), were included in this retrospective interventional case series. The aimed reduction in proptosis was $3-5 \mathrm{~mm}$ and there was no plan to operate externally. Patients with dysthyroid optic neuropathy were excluded. All patients had inactive thyroid eye disease for at least 6 months prior to surgery, as defined by EUGOGO classification [11]. Patients were euthyroid for 6 months prior to surgery. All patients underwent pre and post-operative orbital examinations, which included best-corrected visual acuity (BCVA), ocular motility, Hertel exophthalmometry, 


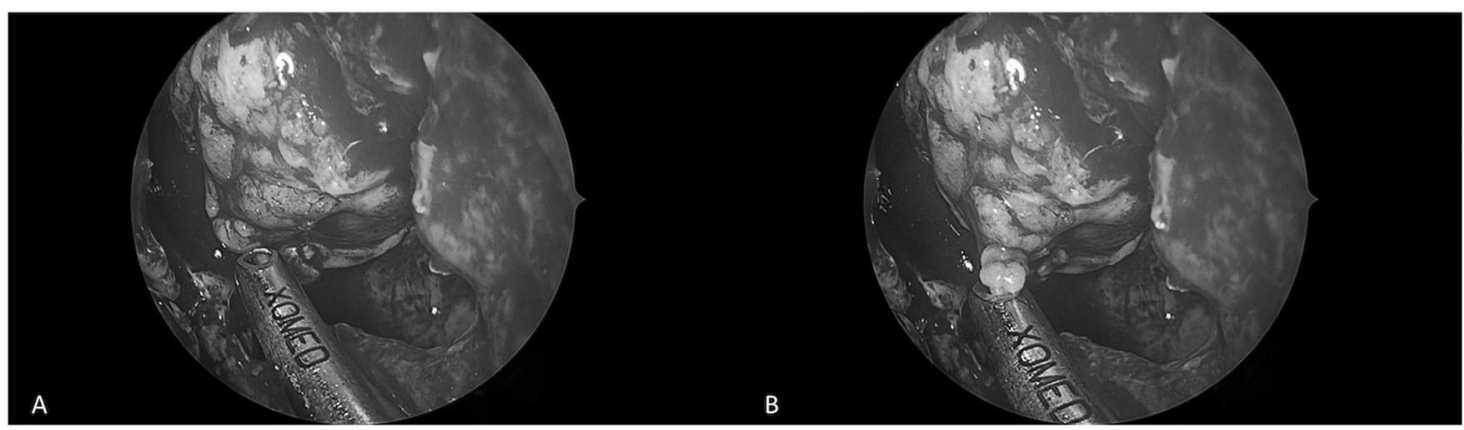

Fig. 1 Endoscopic view of the laryngeal skimmer blade used to excise orbital fat with $\mathbf{a}$ open port and $\mathbf{b}$ orbital fat engaged

slit-lamp biomicroscopy, and fundus examination. All patients were evaluated pre-operatively with computed tomography (CT) of their orbits and paranasal sinuses. Written consent was obtained from all patients before the surgery in accordance with the institution's policies. All patients were followed up for at least 5 months following surgery. The study was approved by the local Institutional Review Board.

\section{Operative technique}

All patients were operated on under general anaesthesia. Anti-platelet and anti-coagulant medication were stopped prior to surgery following consultation with the treating physician, were necessary. Patients received intravenous broad-spectrum antibiotics immediately prior to surgery. The nasal cavity was packed with a solution of cocaine $(2 \%)$ and adrenaline $(1: 10,000)$ on neuropatties prior to sub-mucosal injection with lidocaine $(2 \%)$ and adrenaline (1:80,000). Where a septal deviation prevented sufficient access, a septoplasty was performed. A transnasal sphenoethmoidectomy was performed to expose the medial orbital wall to the orbital apex. The lamina papyracea was removed with a combination of a microcurette, Freer's elevator, and Blakesley forceps to the lesser wing of sphenoid. The periorbita was incised superior and inferior to the medial rectus muscle using a sickle knife to expose the extra-conal orbital fat. Orbital fat was prolapsed into the sinus cavity with the aid of a paediatric ball probe to disrupt the septae between fat lobules. Fat excision was then performed using a laryngeal skimmer blade (Medtronic Inc, Jacksonville, Florida, USA) to excise orbital fat (Fig. 1) on low suction $(50 \mathrm{mmHg})$. The laryngeal skimmer blade used was $22.5 \mathrm{~cm}$ in length with a 15 degree angulation at the distal end and a $3.5 \mathrm{~mm}$ port blade (Fig. 2). Care was taken to avoid area around the medial rectus muscle and its sheath. The titrated fat excision enabled further prolapse of intraconal fat into the sinus. Fat was carefully engaged into the port of the skimmer blade and then cut under direct visualisation of the lumen in short bursts. The volume of orbital fat excised was tailored to each patient and following excision was collected from the suction trap in a syringe to record the volume removed. Post-operatively patients used FESS nasal spray twice daily and were advised to avoid blowing their nose and strenuous physical activity for 2 weeks. They were reviewed at 1 week and 3 months post operatively.

\section{Results}

\section{Patient demographics}

Ninteen patients had surgery whose demographics are summarised in Table 1.

\section{Surgical outcomes}

All patients had endoscopic medial wall decompression, which was combined with endoscopic postero-medial orbital floor removal in 17 patients (89\%). The posterior extent of the medial wall decompression was the minor wing of sphenoid in all cases. In four patients (21\%), a periorbital sling was created once the lamina papyracea was removed. The laryngeal skimmer blade was used to excise orbital fat in all cases and the mean volume of orbital fat excised was $1.45 \pm$ $0.63 \mathrm{ml}$. The mean surgical time was $1.25 \pm 0.47 \mathrm{~h}$. There were no intraoperative complications encountered.

\section{Clinical outcomes}

The changes in globe and lid position are illustrated in Table 2. The mean pre-op BCVA was $0.13 \pm 0.23 \log$ MAR and the mean post op BCVA was $0.14 \pm 0.19 \log$ MAR. The mean reduction in proptosis was $4.5 \pm 1.02 \mathrm{~mm}$. The mean change in vertical palpebral aperture was $-0.84 \pm 0.81 \mathrm{~mm}$. Two patients had improvement in elevation and one improvement in adduction. Five patients subsequently underwent upper lid recession and one underwent lower lid 


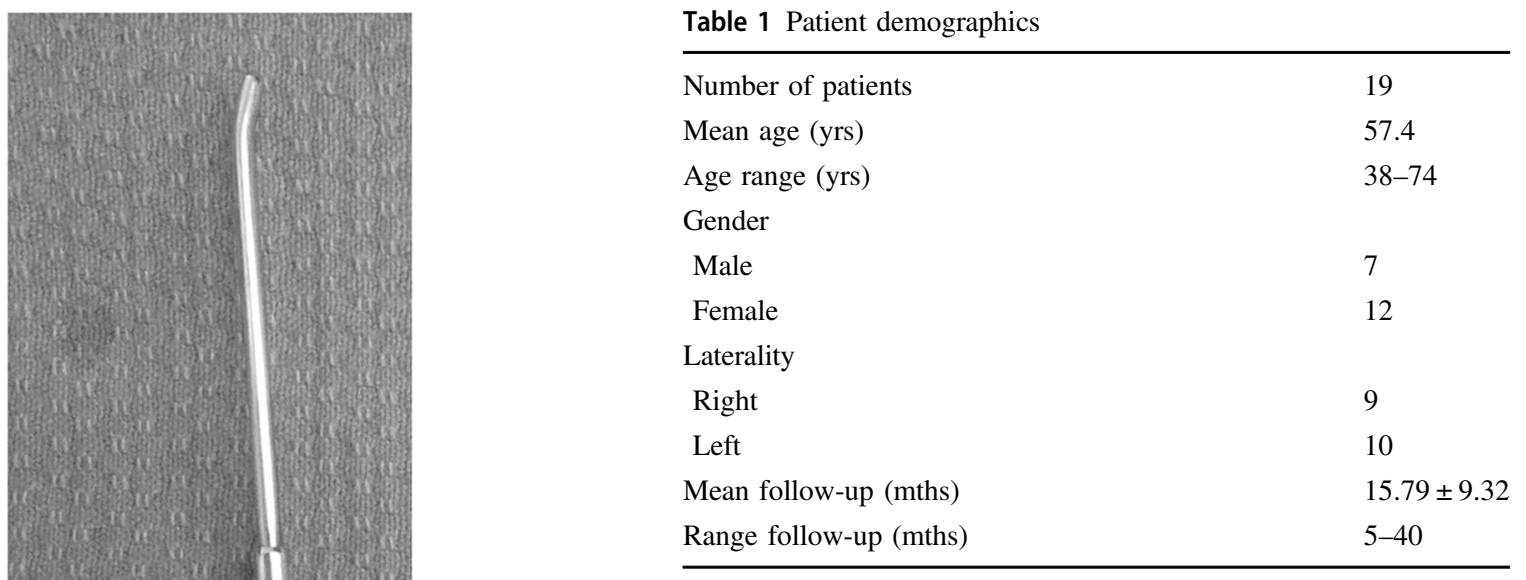

\section{Complications}

Post-operative complications were described in one patient who had transient diplopia which resolved after 6 weeks.

\section{Discussion}

In this study, we describe a technique for orbital fat decompression using a laryngeal skimmer blade and report our outcomes in 19 patients. Orbital fat is commonly encountered when performing orbital decompression for patients with thyroid eye disease. This is most often performed for the management of proptosis, but also in the management of dysthyroid optic neuropathy [12]. Orbital decompression involves bony removal of selected orbital walls and can be combined with orbital fat removal to tailor the amount of decompression and aimed proptosis reduction to the individual patient [13]. Both external and endonasal approaches have been described [5, 13-15]. Some of the challenges handling orbital fat involve working in a confined surgical field, whether that is externally or transnasally, and safely excising orbital fat without damaging muscle or neurovascular structures in close proximity, or indeed causing haemorrhage leading to a compartment syndrome.

Via a transnasal approach, a number of manoeuvres are possible to expose and prolapse orbital fat into the nasal cavity. Once the lamina papyracea has been removed, a central incision in the periorbita, from posterior to anterior can be sufficient to access the orbit, but often the medial rectus muscle will be immediately adjacent to the periorbita and prolapse into the nasal cavity [15]. Alternatively, making parallel incisions above and below the horizontal midline of the periorbita of the medial wall to create a periorbital band or sling can allow prolapse of orbital fat above and below the medial rectus muscle to facilitate fat

excision [16]. 
Table 2 Clinical outcomes

\begin{tabular}{lllr}
\hline & Pre-operative & Post-operative & \multicolumn{1}{c}{ Change } \\
\hline Mean exophthalmometry (mm) & $25.14 \pm 1.25$ & $20 \pm 0$ & $4.52 \pm 1.02$ \\
Mean vertical palpebral aperture (mm) & $11.84 \pm 1.39$ & $11 \pm 1.49$ & $-0.84 \pm 0.81$ \\
Mean intraocular pressure & $19.26 \pm 3.46$ & $18 \pm 2.24$ & $2.53 \pm 2.60$ \\
\hline
\end{tabular}

Prolapsing orbital fat into the nasal cavity is often performed by disrupting the septae between lobules. This may increase the volume of fat being decompressed from the orbit, whether or not it is being excised and moves it to where it can be more safely excised without having to go searching for it with powered instrumentation. Disruption of the orbital septae can be performed with a variety or instruments. In our series, the preferred instrument was a maxillary ostium seeker with a $1.2 \mathrm{~mm}$ diameter ball-shaped end (Karl Storz, Tuttlingen, Germany). The blunt nature of this instrument can minimise the risk of sharp injury to muscle or neurovascular structures.

The laryngeal skimmer blade (Medtronic Inc, Jacksonville, Florida, USA) is a powered instrument used for the excision of lesions in delicate areas of the airway [17]. It is available in a variety of lengths and diameters and the suction can be controlled with the use of a suction regulator. The negative pressure draws tissue into the lumen of the device and once the orbital fat is engaged, the blade, at a fixed cutting rate, excises the tissue in the lumen. This enables controlled excision of orbital fat due to both the high cutting rate and low suction pressure. The cutting blade can operate between speeds of 60-500 revolutions per minute (rpm). In this series, it was used at its maximum operating speed of $500 \mathrm{rpm}$ in order to minimise traction on the fat. One disadvantage is the operator's inability to release the fat once engaged and care must be taken to avoid inadvertent engagement of other tissues into the lumen of the instrument. However, an assistant can manually clamp the suction tubing to prevent further suction of any tissue entrapped within the lumen of the instrument. It should also be noted that the skimmer blade is a longer instrument than required to access the ethmoid sinus region given its length of $22.5 \mathrm{~cm}$. Other techniques to excise orbital fat include use of cutting instruments such as a $3.5 \mathrm{~mm}$ thru-cut Blakesley forceps, although this is a relatively slow process. An alternative device is a manual low suction cutter, which also combines a suction port and a cutting aperture described by Wu et al. [4].

Prolapse of orbital fat into the nasal cavity provides sufficient proptosis reduction in many patients with thyroid eye disease, without the addition of excising the orbital fat, as was described in the original endoscopic medial wall decompression by Kennedy et al. [15]. Endoscopic medial wall decompression without fat excision is thought to reduce proptosis by $\sim 3.5 \mathrm{~mm}[18,19]$. In this cases series, the mean reduction in proptosis was $4.5 \pm 1.02 \mathrm{~mm}$. In our database of 36 patients who underwent endoscopic medial wall decompression without fat excision, the mean reduction in proptosis was $3.6 \pm 0.87 \mathrm{~mm}$.

The results from this study are comparable to techniques of external bony decompression combined with fat excision. Van der Wal et al. [20] report a mean proptosis reduction of $5 \mathrm{~mm}$ in patients who underwent 2-wall decompression combined with fat removal. It is also comparable to techniques of fat decompression without bony decompression as reported by Liao et al. [7] who achieved a mean reduction of proptosis of $4.1 \pm 0.9 \mathrm{~mm}$, but they removed a mean volume of orbital fat of $4.4 \pm 1.2 \mathrm{ml}$. In our series, a smaller volume of fat was excised, $1.45 \pm 0.63 \mathrm{ml}$, achieving a similar effect. Other authors report a much greater degree of proptosis reduction combining an endoscopic medial wall decompression and fat excision, however, they do not report the volume of fat excised [4]. Having the ability to excise fat endoscopically may obviate the need for a combined external approach and may achieve a few millimetres more reduction in proptosis than an endoscopic medial wall decompression alone, in this selected group of patients. Clearly, if an external approach is used in conjunction with an endonasal decompression then fat can also be removed via the external incision potentially obviating the need to do so endonasally.

There are other potential implications for this technique. Excising orbital fat can be performed for biopsy in the context of idiopathic orbital inflammatory disease or to provide visualisation of orbital structures such as the optic nerve or tumours of the mid and posterior orbit, where it may be necessary to remove fat to aide excision of orbital tumours, such as cavernous haemangioma, via an endoscopic route $[21,22]$.

The complications associated with using the laryngeal skimmer blade for orbital fat excision are the same as for any method of orbital fat excision, that of damage to neurovascular structures, inadvertent damage to the extraocular muscles and orbital haemorrhage [12]. The one case of worsening diplopia in this series was attributed to prolapse of the medial rectus muscle following decompression and not directly related to the use of the laryngeal skimmer blade. Maintaining the lumen of the skimmer blade orientated in a superior direction allows direct visualisation of the fat within the lumen and affords greater control whilst minimising inadvertent engagement of the medial rectus muscle or other important 
neurovascular orbital structures. The risk of orbital haemorrhage from orbital fat decompression exists from both and external and endonasal approach. It is the senior author's opinion that management of haemorrhage from orbital fat via an endoscopic route can be done with endonasal suction bipolar forceps with direct visualisation as required and is no more challenging than dealing with bleeding from an external approach. In our series, no cases of orbital haemorrhage were encountered and there was no requirement for endonasal bipolar coagulation.

Some concern exists regarding the risk of diplopia when a combined medial wall and orbital fat excision is not balanced with a lateral orbital decompression [10, 23]. Caution should also be exercised in avoiding overly aggressive fat excision, which might lead to cicatrisation around the medial rectus. However, in our series, only one patient developed post-operative diplopia that resolved by 6 weeks.

\section{Conclusions}

In this study, we describe our experience using a laryngeal skimmer blade as a method of excising orbital fat for orbital decompression in patients with proptosis secondary to Grave's orbitopathy. It can be considered as an adjunctive technique to the orbital surgeon in the endoscopic management of orbital fat and its use could be extrapolated to the excision of orbital fat for other indications.

\section{Summary}

\section{What was known before:}

- Excising orbital fat can be an adjunct to bony decompression in Graves orbitopathy.

- Excision can be performed for visual reasons, in the case of dysthyroid optic neuropathy, or for proptosis.

\section{What this study adds:}

- Orbital fat excision using a laryngeal skimmer blade can be considered as an adjunctive technique to the orbital surgeon for orbital decompression in patients with proptosis secondary to Grave's orbitopathy.

\section{Funding}

There are no financial declarations and the authors report no conflict of interest.

\section{Compliance with ethical standards}

Conflict of interest The authors declare that they have no conflict of interest.

Publisher's note: Springer Nature remains neutral with regard to jurisdictional claims in published maps and institutional affiliations.

\section{References}

1. Gola R, Carreau JP, Faissal A. Adipose tissue of the orbit. Anatomy, and therapeutic deductions. Rev Stomatol Chir maxillofae. 1995;96:123-36.

2. Bahn RS. Graves' ophthalmopathy. N Engl J Med. 2010;362:726-38.

3. Lv Z, Selva D, Yan W, Daniel P, Tu Y, Wu W. Endoscopical orbital fat decompression with medial orbital wall decompression for dysthyroid optic neuropathy. Curr Eye Res. 2016;41:150-8.

4. Wu W, Selva D, Bian Y, Wang X, Sun MT, Kong Q, et al. Endoscopic medial orbital fat decompression for proptosis in type 1 graves orbitopathy. Am J Ophthalmol. 2015;159:277-84.

5. Garrity JA. Orbital lipectomy (fat decompression) for thyroid eye disease: an operation for everyone? Am J Ophthalmol. 2011;151:399-400.

6. Richter DF, Stoff A, Olivari N. Transpalpebral decompression of endocrine ophthalmopathy by intraorbital fat removal (olivari technique): Experience and progression after more than 3000 operations over 20 years. Plast Reconstr Surg. 2007;120:109-23.

7. Liao SL, Huang SW. Correlation of retrobulbar volume change with resected orbital fat volume and proptosis reduction after fatty decompression for graves ophthalmopathy. Am J Ophthalmol. 2011;151:465-69.e1.

8. Leong SC, White PS. Outcomes following surgical decompression for dysthyroid orbitopathy (Graves' disease). Curr Opin Otolaryngol Head Neck Surg. 2010;18:37-43.

9. Chu EA, Miller NR, Lane AP. Selective endoscopic decompression of the orbital apex for dysthyroid optic neuropathy. Laryngoscope 2009;119:1236-40.

10. Kacker A, Kazim M, Murphy M, Trokel S, Close LG. 'Balanced' orbital decompression for severe Graves' orbitopathy: technique with treatment algorithm. Otolaryngol Head Neck Surg. 2003;128:228-35.

11. Bartalena L, Baldeschi L, Dickinson AJ, Eckstein A, KendallTaylor P, Marcocci C, et al. Consensus statement of the European group on Graves' orbitopathy (EUGOGO) on management of Graves' orbitopathy. Thyroid. 2008;18:333-46.

12. Rootman DB. Orbital decompression for thyroid eye disease. Surv Ophthalmol. 2018;63:86-104.

13. Trokel S, Kazim M, Moore S. Orbital fat removal: decompression for Graves orbitopathy. Ophthalmology. 1993;100:674-82.

14. Olivari N. Transpalpebral decompression of endocrine ophthalmopathy (graves' disease) by removal of intraorbital fat: Experience with 147 operations over 5 years. Plast Reconstr Surg. 1991;87:627-41.

15. Kennedy DW, Goodstein ML, Miller NR, Zinreich SJ. Endoscopic transnasal orbital decompression. Arch Otolaryngol Head Neck Surg. 1990;116:275-82.

16. Metson R, Samaha M. Reduction of diplopia following endoscopic orbital decompression: the orbital sling technique. Laryngoscope. 2002;112:1753-7.

17. Ransom ER, Antunes MB, Smith LP, Jacobs IN. Microdebrider resection of acquired subglottic cysts: case series and review of the literature. Int J Pediatr Otorhinolaryngol. 2009;73:1833-6. 
18. Mourits MP, Bijl H, Altea MA, Baldeschi L, Boboridis K, Currò $\mathrm{N}$, et al. Outcome of orbital decompression for disfiguring proptosis in patients with Graves' orbitopathy using various surgical procedures. Br J Ophthalmol. 2009;93:1518-23.

19. Leong SC, Karkos PD, MacEwen CJ, White PS. A systematic review of outcomes following surgical decompression for dysthyroid orbitopathy. Laryngoscope. 2009;119:1106-15.

20. Van der Wal KGH, De Visscher JGAM, Boukes RJ, Smeding B. Surgical treatment of Graves orbitopathy: a modified balanced technique. Int J Oral Maxillofac Surg 2001;30:254-8.
21. Bleier BS, Castelnuovo P, Battaglia P, Turri-Zanoni M, Dallan I, Metson R, et al. Endoscopic endonasal orbital cavernous hemangioma resection: global experience in techniques and outcomes. Int Forum Allergy Rhinol. 2016;6:156-61.

22. Sun MT, Wu W, Yan W, Tu Y, Selva Di. Endoscopic endonasalassisted resection of orbital schwannoma. Ophthal Plast Reconstr Surg. 2017;33(3S):S121-S124.

23. Leone CR, Piest KL, Newman RJ. Medical and lateral wall decompression for thyroid ophthalmopathy. Am J Ophthalmol. 1989;108:160-6. 